\title{
Role of the salt bridge between glutamate 546 and arginine 907 in preservation of autoinhibited form of Apaf-1
}

Raheleh Shakeri, Saman Hosseinkhani, Marek Jan Los, Jamshid Davoodi, Mayur Vilas Jain, Artur Cieslar-Pobuda, Mehrdad Rafat and Sussan Kaboudanian Ardestani

\section{Linköping University Post Print}

\section{Tweet}

N.B.: When citing this work, cite the original article.

Original Publication:

Raheleh Shakeri, Saman Hosseinkhani, Marek Jan Los, Jamshid Davoodi, Mayur Vilas Jain, Artur Cieslar-Pobuda, Mehrdad Rafat and Sussan Kaboudanian Ardestani, Role of the salt bridge between glutamate 546 and arginine 907 in preservation of autoinhibited form of Apaf1, 2015, International Journal of Biological Macromolecules, (81), 370-374.

http://dx.doi.org/10.1016/j.ijbiomac.2015.08.027

Copyright: Elsevier

http://www.elsevier.com/

Postprint available at: Linköping University Electronic Press

http://urn.kb.se/resolve?urn=urn:nbn:se:liu:diva-124522 


\title{
Apaf-1 overexpression induces spontaneous apoptosome formation
}

\author{
Running title: Apaf-1 activity regulation
}

Raheleh Shakeri ${ }^{1}$, Saman Hosseinkhani ${ }^{2}$, Marek J. Los ${ }^{3,4}$, Mayur V. Jain ${ }^{3}$, Artur Cieślar Pobuda $^{3,5}$, Mehrdad Rafat ${ }^{6}$, Jamshid Davoodi ${ }^{1}$, Sussan Kaboudanian Ardestani ${ }^{1}$

\footnotetext{
${ }^{1}$ Institute of Biochemistry and Biophysics, Department of Biochemistry, University of Tehran, Tehran, Iran;

${ }^{2}$ Department of Biochemistry, Faculty of Biological Sciences, Tarbiat Modares University, Tehran, Iran;

${ }^{3}$ Department of Clinical \& Experimental Medicine (IKE), Division of Cell Biology, Integrative Regenerative Med. Center (IGEN), Linköping University, Linköping, Sweden;

${ }^{4}$ Dept. Pathology, Pomeranian Medical University, Szczecin, Poland;

${ }^{5}$ Institute of Automatic Control, Silesian Univ. of Technology, Gliwice, Poland;

${ }^{6}$ Department of Biomedical Engineering, Linköping University, Linköping, Sweden.
}

Correspondence:

\section{Abbreviations:}

KEYWORDS: Apaf-1/Apoptosis/Apoptosome/Caspase-9 


\section{ABSTRACT}

Apaf-1, the key element of apoptotic mitochondrial pathway normally rests in an autoinhibited form inside the cytosol. Typically, cellular stress-triggered cytochrome $c$ release from the mitochondria and its binding to Apaf-1 is required to transform the "closed" (inactive) molecule to open form. WRD-domain of Apaf-1 has a critical role in the preservation of auto-inhibited form, however the underlying mechanism is unclear. It seems the salt bridges between WRD and NOD domain are involved in maintaining the inactive configuration of Apaf-1. At the present study, we have investigated the effect of E546-R907 salt bridge on the maintenance of auto-inhibited form of full length human Apaf-1. E546 residue is mutated to glutamine $(\mathrm{Q})$ and $\operatorname{arginin}(\mathrm{R})$ and the effects of such aa exchange on apoptosome formation and procaspase- 9 activation in the presence or absence of exogenous cytochrome $c$ and dATP is evaluated. Overexpression of wild type, E546Q and E546R Apaf1 in HEK293T cells does not induce apoptosis unlike in HL-60 cancer cell line. In vitro apoptosome formation assay showed that all mutants are cytochrome $c$ and dATP dependent to form apoptosome and activate endogenous procaspase-9 in Apaf-1-knockout MEF cell line. These results suggest that E546 is not a critical residue for preservation of auto-inhibited Apaf-1. Furthermore, exogenous (overexpressed) Apaf-1 can form an in vitro apoptosome in the absence of exogenous cytochrome $c$ and dATP. Exogenous Apaf-1 in the presence of exogenous cytochrome $c$ and dATP processes procaspase-3 in two sites. 


\section{INTRODUCTION}

Apoptosis is essential for development and tissue homeostasis of multi-cellular organisms. Deregulation of this process is associated with various disorders including cancer and neurodegenerative diseases (Akbari-Birgani et al., 2014; Ghavami et al., 2014; Los et al., 1999). Intrinsic apoptotic pathway is activated in response to intracellular stress such as DNA damage, UV and etc, which leads to cytochrome $c$ release from intermembrane space of mitochondria into the cytosol (Green and Reed, 1998). Apaf-1 (apoptotic protease activating factor 1) is a key molecule in the apoptotic intrinsic pathway. It exists in an auto-inhibited form in the cytosol (Zou et al., 1997). In response to intracellular stress, cytochrome $c$ is released from the mitochondria and binds to Apaf-1. This binding induces conformational changes, thus seven Apaf-1 molecules assemble and form a large complex known as apoptosome. Apoptosome is a scaffold for binding and activation of procaspase-9, the initiator caspase in intrinsic apoptotic pathway (Cain, 2003; Kim et al., 2005; TorkzadehMahani et al., 2012; Wurstle et al., 2012).

Apaf-1 molecule consists of three main domains; including CARD (caspase recruitment domain), NOD (nucleotide-binding and oligomerization domain) and WRD (WD40-repeat domain) (Riedl and Salvesen, 2007). Apaf-1 interacts with procaspase-9 by CARD-CARD homotypic interactions (Qin et al., 1999). NOD in the middle of Apaf-1has four sub-domains including NBD (nucleotide-binding domain), HD1 (helical domain 1), WHD (winged-helix domain), and HD2 (helical domainn2) that serve as binding site for nucleotide and apoptosome oligomerization. WRD domain at the c-terminus of Apaf-1 has thirteen WD40 repeats and it composes two $\beta$-propeller including WD1 and WD2 (Reubold et al., 2011; Yu et al., 2005; Yuan et al., 2010). One of the main questions regarding apoptosome formation is how can Apaf-1 preserve its auto-inhibited form in the absence of cytochrome $c$. It has been shown mutant Apaf-1 lacking WRD can activate procaspase-9 in 
the absence of cytochrome $c$ and dATP. Therefore WRD has regulatory function and it prevents from self-assembly of Apaf-1 and apoptosome formation in the absence of cytochrome $c$ (Hu et al., 1998). For many years, it was assumed that WRD domain covers CARD domain of Apaf-1, and prevents from its exposure to interaction with CARD domain of procaspase-9 (Riedl and Salvesen, 2007).

In 2011, the crystal structure of full-length murine Apaf-1 has been determined in the absence of cytochrome $c$ for the first time (Fig. 1A). The structural data reveals that WRD domain is far from CARD domain, and thus WRD and CARD domains do not interact. The cleft between WD1 and WD2 in WRD domain serves as cytochrome $c$ binding site. In the absence of cytochrome $c$, WD1 interacts with NOD and prevents from conformational changes in NOD and CARD domains, which are essential for apoptosome formation. In the absence of WRD, NOD domain is free for conformational changes (Reubold and Eschenburg, 2012; Reubold et al., 2011). The structural data suggests that cytochrome $c$ binding leads to rotation of WD1 upward and it breaks salt bridges between WD1 and NOD domains. After cytochrome $c$ binding, NOD is free for conformational changes, which lead to CARD exposure for interaction with CARD domain of procaspase-9. Three salt bridges between WD1 and NOD domain (K192-D616, E546-R907 and E542-R612) have been identified in the crystal structure of full-length murine Apaf-1, in the absence of cytochrome $c$.

In this study, the importance of the salt bridge between E546 and R907 (Fig. 1B) was evaluated. Our results show that E546Q and E546R Apaf-1 derivates still depend on cytochrome $c$ and dATP for apoptosome formation and procaspase- 9 activation. On the other hand, exogenous Apaf-1 overexpression does not trigger apoptosis in HEK293T cell while it spontaneously induces in vitro apoptosome formation in HEK293T cells. This cell-type dependent Apaf-1 behavior is not fully understood. However, it may relate to the progeny of both cell types, that show very different susceptibility to apoptosis. 


\section{RESULTS}

Overexpression of either wild type Apaf-1 or its derivates E546Q or E546R is not cytotoxic for HEK293T cells

It has been reported that Apaf-1 overexpression can trigger apoptosis in HL-60 cell line (Perkins et al., 1998). Here, we have transfected HEK293T cells with wild type, E546Q-, and E546R Apaf-1. 48 hours upon transfection, the cells were harvested and their viability was assessed by the flow-cytometry-based Po-Pro\&7-AAD method. Our results show that contrary to earlier published data in HL-60 cells, neither the overexpression wild type Apaf-1 nor overexpression or Apaf-1 derivates (E546Q and E546R) does not trigger apoptosis in HEK293T (Fig. 2).

\section{E546Q and E546R Apaf-1 cannot trigger apoptosis in HEK293T cells}

Since the salt bridge between NOD and WD1 has been weakened in E546Q and E546R Apaf-1 to compare wild type Apaf-1, it was expected the mutants Apaf-1 were able to trigger apoptosis without induction. In order to assess apoptosis in response to overexpression of the mutants Apaf-1, all constructs containing wild type and mutants Apaf-1 gene were transfected in HEK293T cells and procaspase-3 cleavage were studied by immunoblotting using anti-caspase-3 antibody. Results showed that E546Q and E546R Apaf-1 cannot trigger apoptosis without cytochrome $c$ release from the mitochondria. It seems broken the salt bridge between E546 with R907 is not required for transformation of Apaf-1 from closed to open form (Fig. 3).

E546Q and E546R mutants Apaf-1 are dependent on cytochrome $c$ and dATP to form apoptosome

Wild-type Apaf-1 in cell lysate can form apoptosome and activate procaspase-9 in the presence of cytochrome $c$ and dATP. In order to assess the ability of E546Q and E546R 
mutants of Apaf-1 to form apoptosome, in vitro apoptosome formation was investigated in the extracts of HEK293T cells transfected with wild type, E546Q, and E546R Apaf-1 plasmids. 48 hours after transfection, cell extracts were prepared and incubated with cytochrome $c$ and dATP to facilitate apoptosome formation. Processing of pro-caspase-3 (a target of caspase-9 that would be activated by apoptosome), was used as a readout of apoptosome formation. As indicated in figure 3, no procaspase-3 processing could be detected neither in samples containing wild-type Apaf-1, nor in samples containing Apaf-1 mutants. Tubulin was used as an indicator for equal protein loading.

We have next directly determined the capsase- 9 activation by using colorimetric assay. HEK293T cell extracts containing wild-type, E546Q, and E546R Apaf-1 were evaluated for LEHDase activity. Surprisingly, we detected LEHDase activity in HEK293T cell extracts containing wild type, E546Q and E546R Apaf-1, both in the presence and absence of exogenous cytochrome $c$ and dATP (Fig. 4). Furthermore, caspase-9 activation in the absence of cytochrome $c$ and dATP was confirmed by Western blot analysis of procaspase-9, which was consistent with data from caspase-9 assay (Fig. 5). It maybe these unexpected results were due to endogenous Apaf-1 in HEK293T cell extract. To explain this ambiguity, in vitro apoptosome formation and procaspase-9 activation were performed in Apaf-1-knockout MEF cell line, which lacks endogenous Apaf-1 expression. Results showed that cell extracts containing E546Q and E546R Apaf-1 are able to activate procaspase-9 to a similar degree as wild-type Apaf-1, only in the presence of cytochrome $c$ and dATP (Fig.6).

Procaspase-3 cleavage pattern in HEK293T cell extracts containing exogenous Apaf-1 is different in the presence and absence of exogenous cytochrome c and dATP

One of the components of apoptosome is procaspase-3, which is processed and activated by caspase-9 inside the apoptosome. We have thus determined procaspase- 3 cleavage by in vitro activated caspase-9, in HEK293T cell transfected either with wild-type Apaf-1 or its 
derivates E546Q and E546R. The assay was done both in the presence, and in the absence of cytochrome $c$ and dATP. The assay samples were then analyzed by immunoblotting using anti-caspase-3 antibody. As shown in figure 7, procaspase-3 cleavage pattern in HEK293T cell extracts containing exogenous Apaf-1 in the presence or absence of exogenous cytochrome $c$ and dATP differed. In the absence of exogenous cytochrome $c$ and dATP, a 17 $\mathrm{kDa}$ band (large subunit of caspase-3) could be detected. While in the presence of exogenous cytochrome $c$ and dATP, as well as large subunit of caspase-3, another band around $31 \mathrm{kDa}$ also appears. 


\section{DISCUSSION}

Apaf-1 is the key player in the mitochondrial apoptotic pathway. It is normally found in the cytosol in its auto-inhibited (closed) form (Zou et al., 1997). Crystal structure of full-length murine Apaf-1, in the absence of cytochrome $c$ at $3.55 \mathrm{~A}^{\circ}$ resolution has recently been determined (Reubold et al., 2011). It has been demonstrated earlier, that the role of WRDdomain at the c-terminal of Apaf-1 is to stabilize it in its auto-inhibited form, because mutant of Apaf-1 lacking WRD-domain, spontaneously assembles and form apoptosome, even in the absence of cytochrome $c$ (Hu et al., 1998; Srinivasula et al., 1998). So far, it is not known, how does full length Apaf-1 keep itself in auto-inhibited form, in the absence of cytochrome c. Based on Apaf-1's crystal structure it has been determined that cytochrome $c$ binds into cleft between WD1 and WD2, and that leads to WD1 rotation upward and subsequent release of inhibitory effect of WD1 on NOD domain. This rotation induces conformational changes and exposure of CARD domain (Reubold et al., 2011). In mutant Apaf-1, lacking WRD, the inhibitory effect of WRD has removed and NOD domain is free for conformational changes and oligomerization (Reubold and Eschenburg, 2012). Before determination of the crystal structure of full length Apaf-1, it was proposed that WRD domain preserved closed form of Apaf-1 by covering CARD domain (Riedl and Salvesen, 2007). But the crystal structure has shown that WRD domain is very far from CARD domain. So it cannot interact with CARD domain and cover it. It has suggested that WRD interactions with NOD domain prevent CARD domain exposure in the absence of cytochrome $c$ (Reubold and Eschenburg, 2012). It has been shown that salt bridges between WD1 and NOD domains exist in the crystal structure (Reubold et al., 2011). The role of these salt bridges for preservation of autoinhibited Apaf-1 is still unclear. In order to weaken the interactions between WD1 and NOD domains, E546, involving a salt bridge with R907, we have prepared mutants E546Q (an uncharged residue) and E546R (a positive charge residue). Next, wild-type, E546Q and 
E546R Apaf-1 were overexpressed in HEK293T cells. It has been reported that overexpression of full length Apaf-1 triggers apoptosis in human myeloid leukemia HL-60 cells (Perkins et al., 1998). On the other hand, resistance of human leukemia cell lines such as K562, CEM and CEM/VLB 100 is due to deficiency in Apaf-1 molecule. Apaf-1 gene transfection restores the sensitivity of resistant leukemic cells to apoptosis (Jia et al., 2001).

As indicated in figures 2 and 3, a higher intracellular level of Apaf-1 resulting from its overexpression, does not trigger apoptosis in HEK293T cells unlike the human myeloid leukemia HL-60 cells (Perkins et al., 1998). Overexpression of mutants (E546Q and E546R) Apaf-1 in HEK293T cells was similar to wild-type Apaf-1. Thus, likely the level of Apaf-1 is not rate limiting step in the apoptotic mitochondrial pathway of in HEK293T cell line.

The difference in the sensitivity to Apaf-1 overexpression between HL-60 and HEK293T, may be dependent on the cell type. HEK293T cells are a transformed human cell line while HL-60 is a cancer cell line (Graham et al., 1977; Thomas and Smart, 2005). Cancer cell lines are able to produce more ATP using the Warburg effect (Qian et al., 2014). Thus, higher level of ATP may facilitate activation of abundant/overexpressed Apaf-1 and apoptosome formation (Perkins et al., 1998). The difference in apoptotic sensitivity towards Apaf-1 overexpression, may also be explained based on the behavior of healthy counterparts of both cell types. HL-60 cells behave similarly to neutrophiles, that are very sensitive to apoptosis, whereas HEK293T originate from kidney cells that are markedly more resistant towards apoptosis.

To further characterize the ability of E546Q and E546R in apoptosome formation, HEK293T cell extracts expressiong wild-type and mutants of Apaf-1 were assessed for in vitro apoptosome formation and caspase-9 activation. Surprisingly, HEK293T cell extracts containing overexpressed Apaf-1 activated procaspase-9 in the presence and absence of both 
cytochrome $c$ and dATP, which implies that apoptosome oligomerizes independently of exogenous cytochrome $c$ and dATP if Apaf-1 is abundant (Fig. 4 and 5).

The afore mentioned observation may have been influenced by intrinsic Apaf-1. Thus we have performed in vitro apoptosome formation assay in murine Apaf-1 deficient cell line (immortalized MEF). Caspase-9 assay showed that E546Q and E546R Apaf-1 were dependent on cytochrome $c$ and dATP for caspase-9 activation such as wild type Apaf-1 (Fig. $6)$.

Based on our results, we propose that E546 is not critical residue to preserve the autoinhibited form of human Apaf-1. Moreover, our data suggests that the distance between E546 and R907 is to high for salt bridge to form. On the other hand, the salt bridge has been demonstrated in the crystal structure of full length murine Apaf-1 that has $87.3 \%$ sequence identity with human full length Apaf-1 (Reubold et al., 2011). Since in this study, the salt bridge between E546 and R907 has been broken in the human full length Apaf-1 and this event did not effect on Apaf-1 function, it may be due to different molecular orientation of the human full length Apaf-1 relative to its murine ortholog. Therefore, it may be suggested that disruption of two other suggested salt bridges in Apaf-1 by site directed mutagenesis on apoptosome formation is worth pursuing experimentally.

To confirm caspase-9 activation in HEK293T cell extracts containing exogenous Apaf-1, procaspase-9 processing was examined. The results of immunobloting were consistent with caspase-9 assay (Fig. 5). The caspase-9 activity detected in the absence of cytochrome $c$ and dATP may be explained by exogenous Apaf-1 oligomerization occurring in the presence of low level of endogenous cytochrome $c$ in cell lysates. It should be noted that the level of endogenous cytochrome $c$ in all prepared HEK293T cytosolic extracts were the same (Fig. 7). Endogenous Apaf-1 cannot form apoptosome in the absence of exogenous cytochrome $c$ and dATP in spite of exogenous Apaf-1. On the other hand, it may be proposed 
that the folding of exogenous Apaf-1 is different from endogenous Apaf-1 in HEK293T cells, and exogenous Apaf-1 has partially open form that only needs incubation at $37^{\circ} \mathrm{C}$ for transformation and in vitro apoptosome formation without any additional inducer like cytochrome $c$. Moreover, it is suggested that preservation of closed form of endogenous Apaf-1 needs other cofactors that release after cytochrome $c$ binding and apoptosome formation. But under condition where Apaf-1 is overexpressed, the cell does not have enough amounts of these cofactors for maintenance of closed form of exogenous Apaf-1. As noted the cancer cells produce more ATP using upregulated aerobic glycolysis (Qian et al., 2014). That may explain why exogenous Apaf-1 can trigger apoptosis in HL-60 cancer line in the absence of cytochrome $c$ release. But exogenous Apaf-1 overexpression cannot trigger apoptosis in HEK293T cells whereas can forms apoptosome in cell lysate after incubation at $37^{\circ} \mathrm{C}$.

It has been reported that apoptosome, in addition to caspase-9, contains fully processed caspase-3 and caspase-7 (Bratton et al., 2001). In order to confirm in vitro apoptosome formation in HEK293T cell extracts containing exogenous wild type and mutants full length Apaf-1 in the absence of exogenous cytochrome $c$ and dATP, procaspase3 processing were assessed by Western blot with the monoclonal anti-caspase-3 antibody. Surprisingly, procaspase-3 cleavage pattern in HEK293T cell extracts containing exogenous full length Apaf-1 in the presence and absence of cytochrome $c$ and dATP was different. In the absence of exogenous cytochrome $c$ and dATP, anti-caspase- 3 antibody recognizes one band around $17 \mathrm{kDa}$ (p17) but in the presence of exogenous cytochrome $c$ and dATP recognizes two cleaved caspase- 3 which one is a $17 \mathrm{kDa}(\mathrm{p} 17)$ and second is a $31 \mathrm{kDa}(\mathrm{p} 31)$ (Fig. 7). It seems that cytochrome $c$ and dATP addition to exogenous Apaf-1 in cell lysate leads to different cleavages in procaspase-3. The number of formed apoptosomes in the presence or absence of exogenous cytochrome $c$ and dATP are different and leads to different 
rate in procaspase-3 processing. It may be suggested, in the presence of exogenous cytochrome $c$ and dATP, the numbers of apoptosomes are more and lead to distribute procaspase- 9 in increased apoptosome and decreases procaspase- 9 processing efficiency and procaspase-3 processing.

In conclusion, based on the results presented in this manuscript, the E546 residue is not critical for preservation of auto-inhibited form of full length human Apaf-1. Furthermore, Apaf-1 overexpression does not trigger apoptosis in HEK293T cell line, however it can form in vitro apoptosome, independently of exogenous cytochrome $c$ and dATP. 


\section{MATERIALS AND METHODS}

Site directed mutagenesis of full-length human Apaf-1: The Quick change site-directed mutagenesis method was used to make E546Q and E546R mutants from pcDNA3-Apaf-1XL-Myc (gift from Dr. Gabriel Nunez) (Wang and Malcolm, 1999). The nucleotide sequences of the plasmids were confirmed by nucleotide full sequencing (an automatic sequencer Macrogen).

Transfection, in vitro activation of apoptosome and immunoblotting: Apaf-1-knockout murine embryonic fibroblasts (Apaf-1-knockout MEF) and human embryonic kidney 293 (HEK293T) cells were transfected with wild type, E546Q and E546R Apaf-1 plasmids when $80 \%$ confluent using Polyethylenimine reagent (Polysciences, Inc). $48 \mathrm{~h}$ after transfection, cells were lysed in buffer A (20 mM HEPES-KOH (pH 7.5), $10 \mathrm{mM} \mathrm{KCl,} 1.5 \mathrm{mM} \mathrm{MgCl} 2,1$ mM EDTA, 1mM DTT and $250 \mathrm{mM}$ Sucrose ) containing protease inhibitor cocktail (Sigma) and passed 10 times through a 30-gauge needle. Following centrifugation at $15000 \times \mathrm{g}$ for 30 min at $4^{\circ} \mathrm{C}$, cytosolic extract (supernatants) were collected and determined the protein concentration using Bradford method. For in vitro activation of apoptosome, $100 \mu \mathrm{g}$ of cytosolic extract were combined with or without $20 \mu \mathrm{g} / \mathrm{ml}$ of cytochrome $c$ (Sigma) and $1 \mathrm{mM}$ of dATP (Sigma) for $30 \mathrm{~min}$ at $37^{\circ} \mathrm{C}$ and used for immunoblotting with relevant antibodies (McStay and Green, 2014). Rabbit anti-Apaf-1, mouse anti-Myc, mouse anticytochrome $c$ and mouse anti-caspase-3 antibodies were obtained from Abcam. Mouse anticaspase-9 was obtained from Santa Cruz Biotech. The secondary antibody anti-rabbit HRPconjugate were obtained from Bio-RAD and anti-murine HRP-conjugate was from Sigma Aldrich. 
Caspase-9 assay: Cytosolic extracts of the indicated plasmids in the presence or absence of cytochrome $c$ and dATP were assayed for caspase-9 activity using synthetic chromogenic substrate Ac-LEHD-pNA (Sigma-Aldrich) (Denault and Salvesen, 2003). $100 \mu \mathrm{g}$ of cytosolic extract were assayed in $50 \mu \mathrm{l}$ of $2 \mathrm{x}$ caspase assay buffer $(20 \mathrm{mM}$ HEPES pH 7.2, 200mM $\mathrm{NaCl}, 20 \%$ Sucrose, $0.2 \%$ CHAPS, $2 \mathrm{mM}$ EDTA and 40mM $\beta$-mercaptoethanol). The reaction was started with addition of $0.2 \mathrm{mM}$ Ac-LEHD- $\rho \mathrm{NA}$ and $\rho \mathrm{NA}$ released was measured at various times following the start of the reaction using plate reader machine (BioTek) at $405 \mathrm{~nm}$.

Po-Pro and 7-AAD cell death assay: HEK293T cells were seeded in each well of 12-well plates. After $16 \mathrm{~h}$, cells were transiently transfected with indicated plasmids. $24 \mathrm{~h}$ and $48 \mathrm{~h}$ after transfection, cells were collected and washed with PBS for 3 times. The cells were resuspended in PBS including Po-Pro and 7-AAD dyes on ice for $30 \mathrm{~min}$. The percentage of apoptotic cells was analyzed using flow cytometry machine (Gallios, Beckman Coulter Inc). Flow cytometry results were analyzed using Kaluza analysis software (Beckman Coulter Inc.).

Conflict of Interest: Authors declare no conflict of interest.

Acknowledgements: MJL kindly acknowledges the core/startup support from Linkoping University, from Integrative Regenerative Medicine Center (IGEN), from Cancerfonden (2013/391), and from VR-NanoVision (K2012-99X -22325-01-5). 


\section{REFERENCES}

Akbari-Birgani, S., Hosseinkhani, S., Mollamohamadi, S., and Baharvand, H. (2014). Delay in apoptosome formation attenuates apoptosis in mouse embryonic stem cell differentiation. The Journal of biological chemistry 289, 16905-16913.

Bratton, S.B., Walker, G., Srinivasula, S.M., Sun, X.M., Butterworth, M., Alnemri, E.S., and Cohen, G.M. (2001). Recruitment, activation and retention of caspases-9 and -3 by Apaf-1 apoptosome and associated XIAP complexes. The EMBO journal 20, 998-1009.

Cain, K. (2003). Chemical-induced apoptosis: formation of the Apaf-1 apoptosome. Drug metabolism reviews 35, 337-363.

Denault, J.B., and Salvesen, G.S. (2003). Expression, purification, and characterization of caspases. Current protocols in protein science / editorial board, John E Coligan [et al] Chapter 21, Unit 2113. Ghavami, S., Shojaei, S., Yeganeh, B., Ande, S.R., Jangamreddy, J.R., Mehrpour, M., Christoffersson, J., Chaabane, W., Moghadam, A.R., Kashani, H.H., et al. (2014). Autophagy and apoptosis dysfunction in neurodegenerative disorders. Progress in neurobiology 112, 24-49.

Graham, F.L., Smiley, J., Russell, W.C., and Nairn, R. (1977). Characteristics of a human cell line transformed by DNA from human adenovirus type 5 . The Journal of general virology 36, 59-74. Green, D.R., and Reed, J.C. (1998). Mitochondria and apoptosis. Science 281, 1309-1312.

Hu, Y., Ding, L., Spencer, D.M., and Nunez, G. (1998). WD-40 repeat region regulates Apaf-1 selfassociation and procaspase-9 activation. The Journal of biological chemistry 273, 33489-33494. Jia, L., Srinivasula, S.M., Liu, F.T., Newland, A.C., Fernandes-Alnemri, T., Alnemri, E.S., and Kelsey, S.M. (2001). Apaf-1 protein deficiency confers resistance to cytochrome c-dependent apoptosis in human leukemic cells. Blood 98, 414-421.

Kim, H.E., Du, F., Fang, M., and Wang, X. (2005). Formation of apoptosome is initiated by cytochrome c-induced dATP hydrolysis and subsequent nucleotide exchange on Apaf-1. Proceedings of the National Academy of Sciences of the United States of America 102, 17545-17550.

Los, M., Wesselborg, S., and Schulze-Osthoff, K. (1999). The role of caspases in development, immunity, and apoptotic signal transduction: lessons from knockout mice. Immunity 10, 629-639. McStay, G.P., and Green, D.R. (2014). Preparation of cytosolic extracts and activation of caspases by cytochrome c. Cold Spring Harbor protocols 2014, 778-782.

Perkins, C., Kim, C.N., Fang, G., and Bhalla, K.N. (1998). Overexpression of Apaf-1 promotes apoptosis of untreated and paclitaxel- or etoposide-treated HL-60 cells. Cancer research 58, 4561-4566.

Qian, Y., Wang, X., Liu, Y., Li, Y., Colvin, R.A., Tong, L., Wu, S., and Chen, X. (2014). Extracellular ATP is internalized by macropinocytosis and induces intracellular ATP increase and drug resistance in cancer cells. Cancer letters 351, 242-251. Qin, H., Srinivasula, S.M., Wu, G., Fernandes-Alnemri, T., Alnemri, E.S., and Shi, Y. (1999). Structural basis of procaspase-9 recruitment by the apoptotic protease-activating factor 1 . Nature 399, 549-

557.

Reubold, T.F., and Eschenburg, S. (2012). A molecular view on signal transduction by the apoptosome. Cellular signalling 24, 1420-1425.

Reubold, T.F., Wohlgemuth, S., and Eschenburg, S. (2011). Crystal structure of full-length Apaf-1: how the death signal is relayed in the mitochondrial pathway of apoptosis. Structure 19, 1074-1083. Riedl, S.J., and Salvesen, G.S. (2007). The apoptosome: signalling platform of cell death. Nature reviews Molecular cell biology 8, 405-413.

Srinivasula, S.M., Ahmad, M., Fernandes-Alnemri, T., and Alnemri, E.S. (1998). Autoactivation of procaspase-9 by Apaf-1-mediated oligomerization. Molecular cell 1, 949-957.

Thomas, P., and Smart, T.G. (2005). HEK293T cell line: a vehicle for the expression of recombinant proteins. Journal of pharmacological and toxicological methods 51, 187-200.

Torkzadeh-Mahani, M., Ataei, F., Nikkhah, M., and Hosseinkhani, S. (2012). Design and development of a whole-cell luminescent biosensor for detection of early-stage of apoptosis. Biosensors \& bioelectronics 38, 362-368. 
Wang, W., and Malcolm, B.A. (1999). Two-stage PCR protocol allowing introduction of multiple mutations, deletions and insertions using QuikChange Site-Directed Mutagenesis. BioTechniques 26,

680-682.

Wurstle, M.L., Laussmann, M.A., and Rehm, M. (2012). The central role of initiator caspase-9 in apoptosis signal transduction and the regulation of its activation and activity on the apoptosome.

Experimental cell research 318, 1213-1220.

Yu, X., Acehan, D., Menetret, J.F., Booth, C.R., Ludtke, S.J., Riedl, S.J., Shi, Y., Wang, X., and Akey,

C.W. (2005). A structure of the human apoptosome at $12.8 \mathrm{~A}$ resolution provides insights into this

cell death platform. Structure 13, 1725-1735.

Yuan, S., Yu, X., Topf, M., Ludtke, S.J., Wang, X., and Akey, C.W. (2010). Structure of an apoptosomeprocaspase-9 CARD complex. Structure 18, 571-583.

Zou, H., Henzel, W.J., Liu, X., Lutschg, A., and Wang, X. (1997). Apaf-1, a human protein homologous to C. elegans CED-4, participates in cytochrome c-dependent activation of caspase-3. Cell 90, 405- 


\section{FIGURES}

A

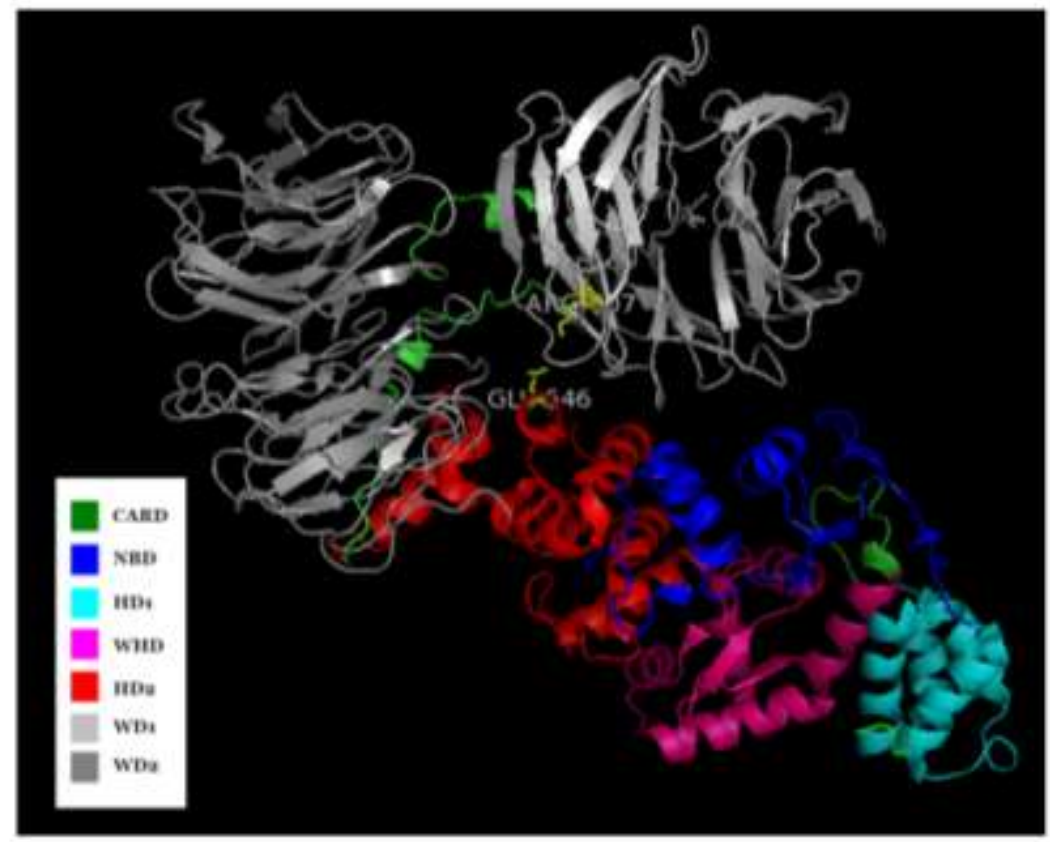

B

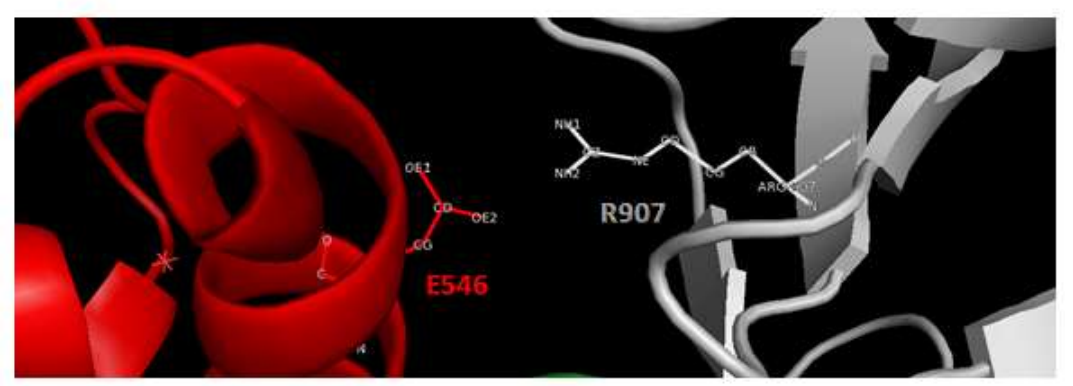

Figure 1. (A) Crystal structure of full length murine Apaf-1 (PDB ID: 3SFZ). (B) E546 in HD2 has salt bridge with R907 in WD1. The visualization is done by PyMOL software. 


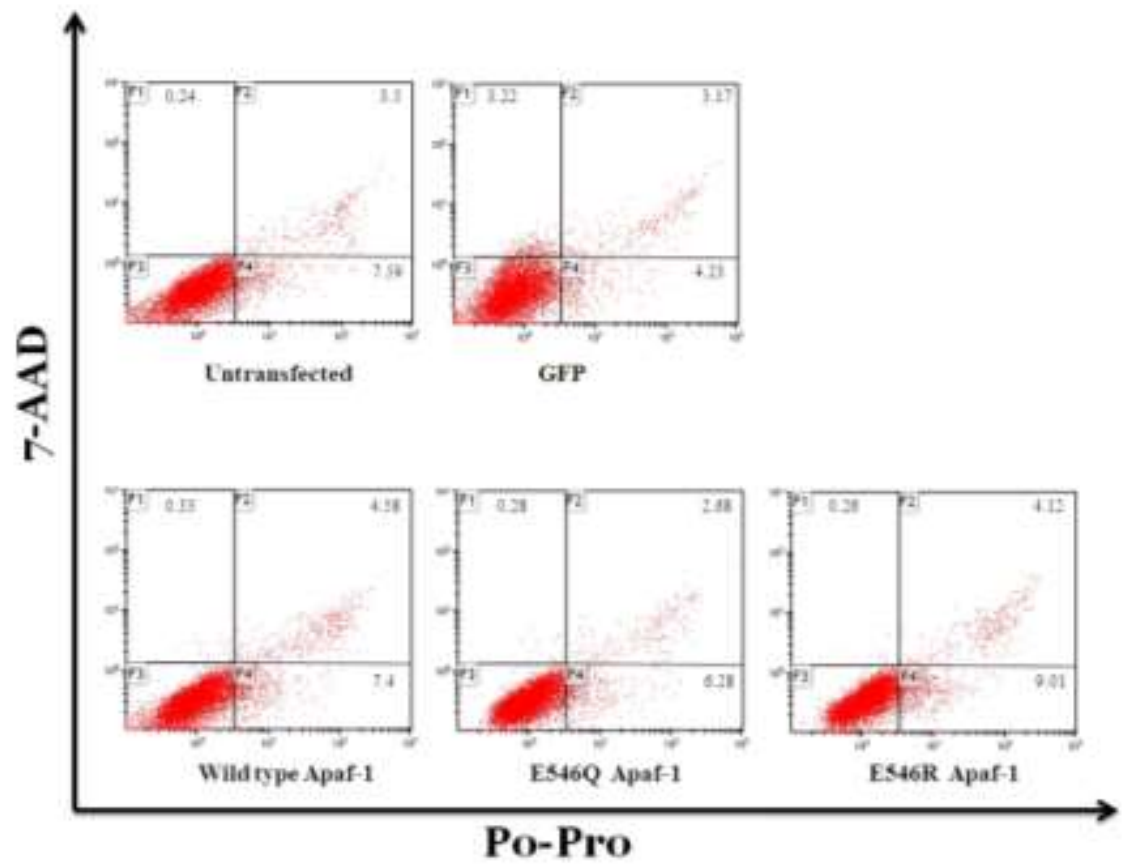

Figure 2. Cell viability assessment in response to Apaf-1 overexpression in HEK293T cells. The cells were transiently transfected with GFP, wild type, E546Q and E546R mutants Apaf-1 plasmids then harvested 48 hours upon transfection and cell viability were investigated by Po-Pro and \&-AAD assay. Viable cells (Po-Pro-/7AAD-), early apoptotic cells (Po-Pro+/7AAD-), necrotic cells (Po-Pro-/7AAD+), and dead cells (Po-Pro+/7AAD+) are located in the lower left, lower right, upper left, and upper right quadrants, respectively. Numbers in each quadrant represent the percentage of cells.

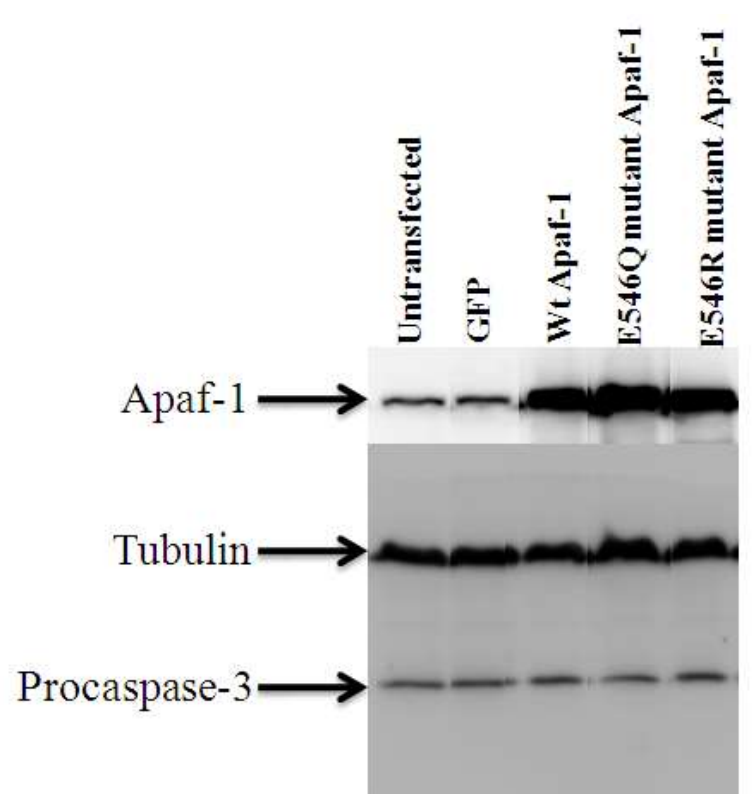


Figure 3. Assessment of procaspase-3 processing as a sign of apoptosis in response to Apaf-1 overexpression. HEK293T cells transfected with indicated plasmids were lysed and assessed by western blot with anti-caspase-3, anti-Apaf- 1 and anti-tubulin antibodies.
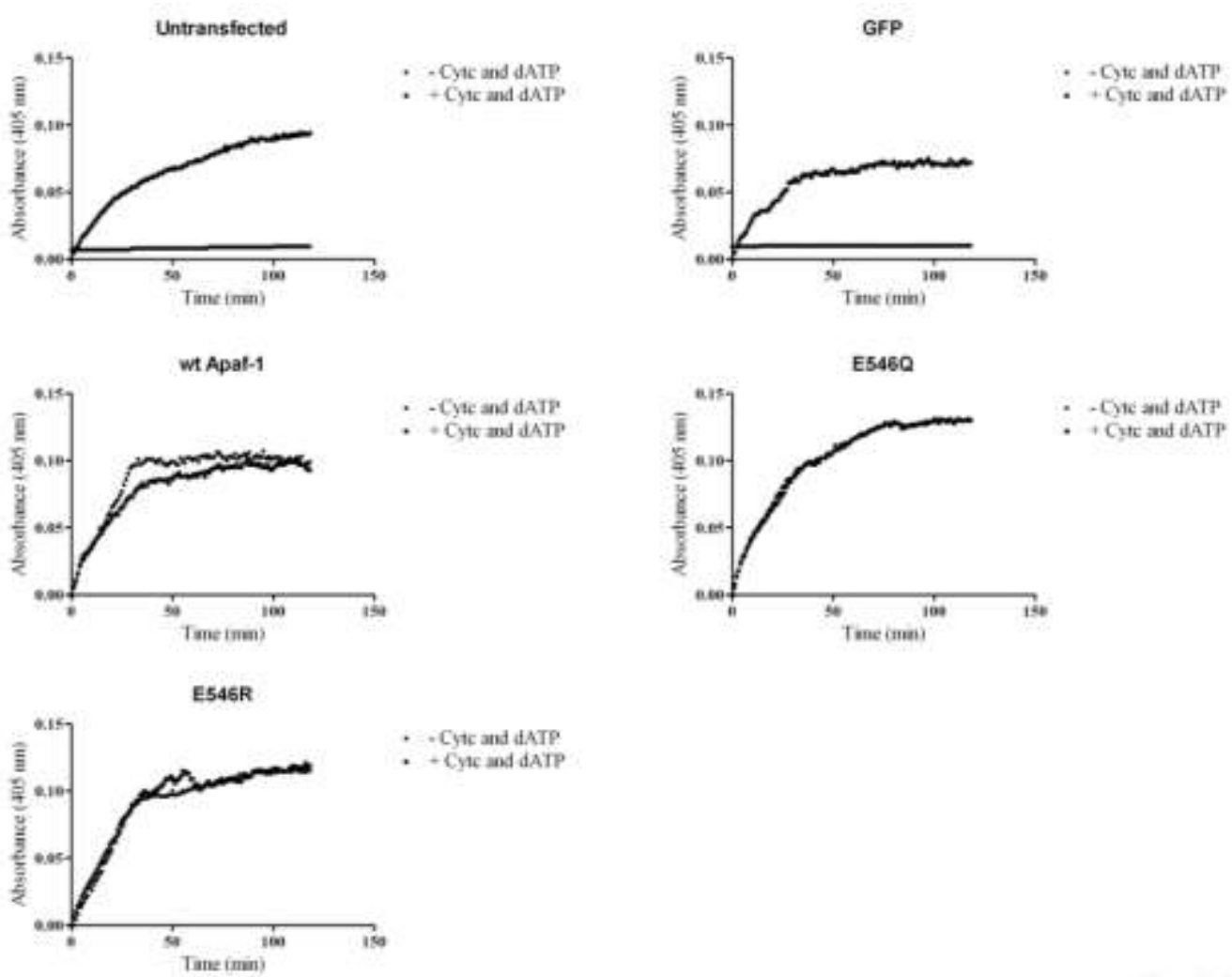

Figure 4. Time course of endogenous caspase-9 activity after in vitro apoptosome formation in HEK293T cell lysate. The cells were transiently transfected with GFP, wtApaf-1, E546Q and E546R mutants Apaf-1 plasmids. Lysate from untransfected and transfected were divided into two equal parts and one part were treated with cytochrome $c$ and $\mathrm{dATP}$, then incubated at $37^{\circ} \mathrm{C}$ for $30 \mathrm{~min}$ for apoptosome formation. Lysate were analyzed for caspase-9 activity using Ac-LEHD- $\rho$ NA chromogenic substrate at $405 \mathrm{~nm}$. 


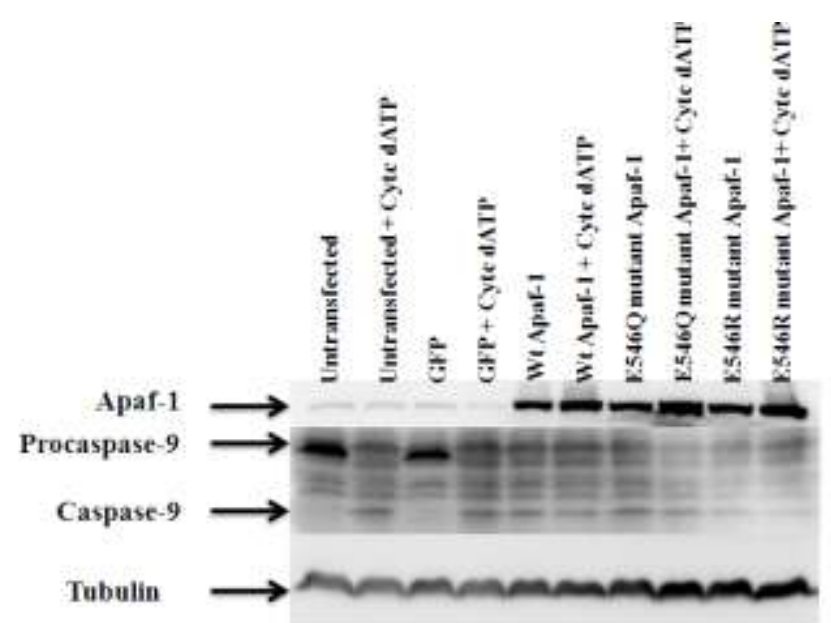

Figure 5. Procaspase-9 processing after in vitro apoptosome formation in HEK293T cell lysate. HEK293T cells were transiently transfected with GFP, wtApaf-1, E546Q and E546R mutants Apaf-1 plasmids. Lysate from untransfected and transfected were divided into two equal parts and one part were treated with cytochrome $c$ and dATP, then incubated at $37^{\circ} \mathrm{C}$ for $30 \mathrm{~min}$ for apoptosome formation. Lysate were analyzed by western blot for procaspase- 9 processing.
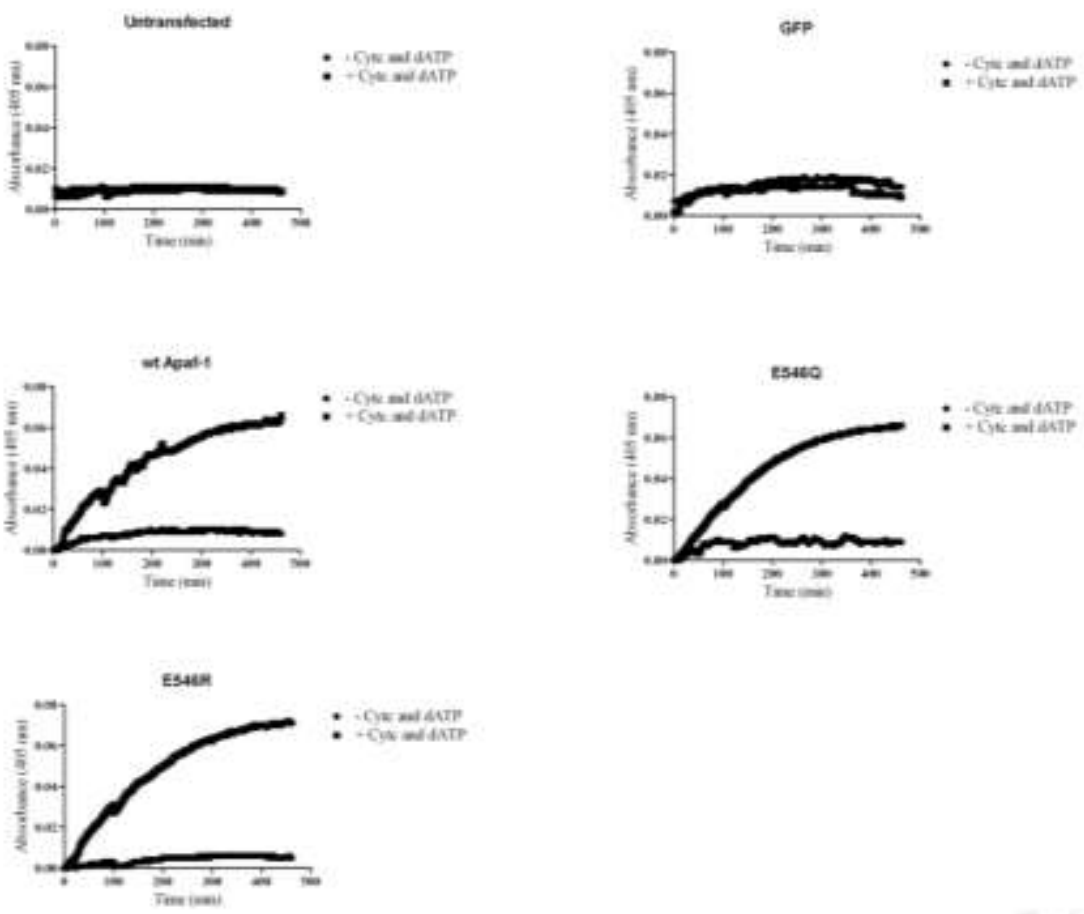
Figure 6. Time course of endogenous caspase-9 activity after in vitro apoptosome formation in Apaf-1-knockout MEF cell lysate. Apaf-1-knockout MEF cells were transiently transfected with GFP, wtApaf-1, E546Q and E546R mutants Apaf-1 plasmids. Lysate from untransfected and transfected were divided into two equal parts and one part were treated with cytochrome $c$ and dATP, and then incubated at $37^{\circ} \mathrm{C}$ for $30 \mathrm{~min}$ for apoptosome formation. Lysate were analyzed for caspase-9 activity using Ac-LEHD- $\rho N A$ chromogenic substrate at $405 \mathrm{~nm}$.

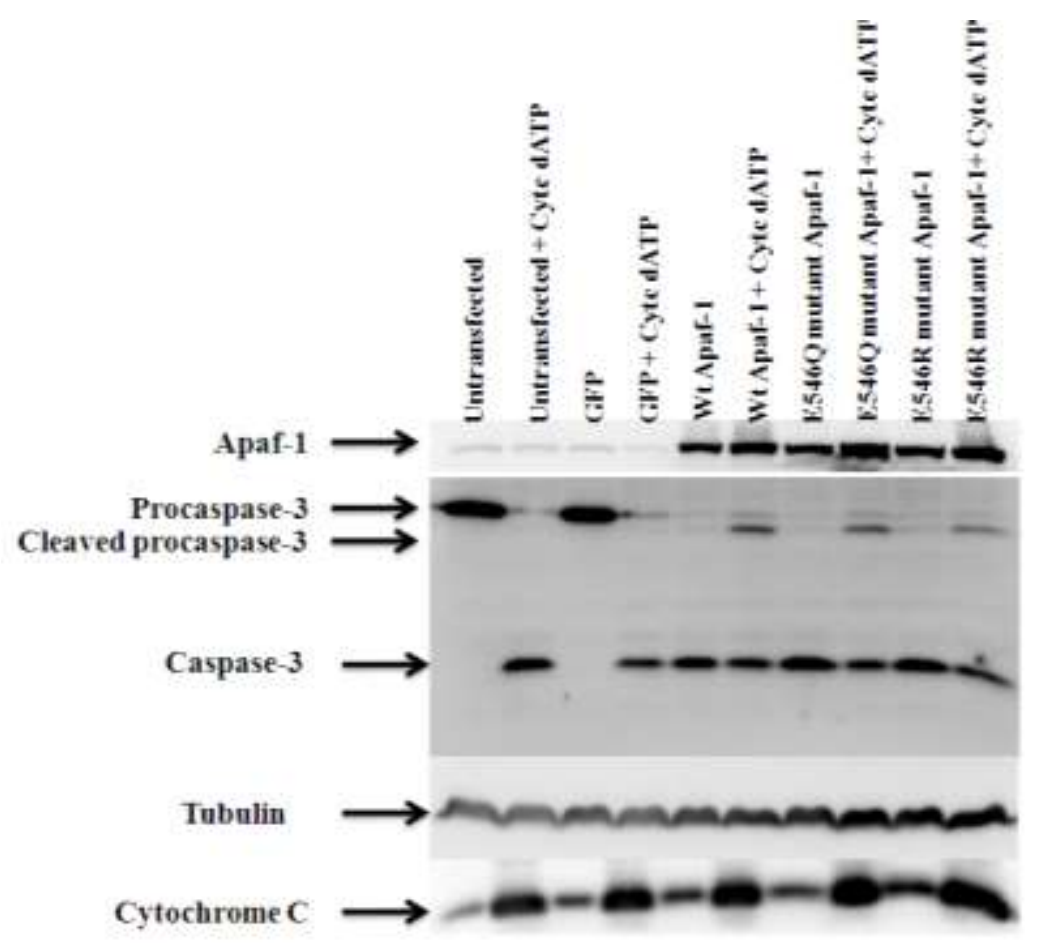

Figure 7. Cleavage of procaspase-3 after in vitro apoptosome formation in HEK293T cell lysate. HEK293T cells were transiently transfected with GFP, wtApaf-1, E546Q and E546R mutants Apaf-1 plasmids. Lysate from untransfected and transfected were divided into two equal parts and one part were treated with cytochrome $c$ and dATP, then incubated at $37^{\circ} \mathrm{C}$ for $30 \mathrm{~min}$ for apoptosome formation. Lysate were analyzed by Western blot using anti-Apaf-1, anti-caspase-3, anti-tubulin and anti-cytochrome c antibodies. 